\title{
Gap Fluctuations in Inhomogeneous Superconductors
}

\author{
Julia S. Meyer ${ }^{1,2}$ and B. D. Simons ${ }^{1}$ \\ 1 Cavendish Laboratory, Madingley Road, Cambridge CB3 0HE, UK \\ 2 Theoretische Physik III, Ruhr-Universität Bochum, 44780 Bochum, Germany
}

(November 1, 2018)

\begin{abstract}
Spatial fluctuations of the effective pairing interaction between electrons in a superconductor induce variations of the order parameter which in turn lead to significant changes in the density of states. In addition to an overall reduction of the quasi-particle energy gap, theory suggests that mesoscopic fluctuations of the impurity potential induce localised tail states below the mean-field gap edge. Using a field theoretic approach, we elucidate the nature of the states in the 'sub-gap' region. Specifically, we show that these states are associated with replica symmetry broken instanton solutions of the mean-field equations.
\end{abstract}

PACS numbers: 74.40.+k 74.62.Dh 74.80.-g

\section{INTRODUCTION}

The density of states (DoS) of a bulk s-wave superconductor exhibits a quasi-particle energy gap and a singularity at the gap edge. While the gap is robust with respect to the addition of non-magnetic impurities (Anderson theorem [1]), the integrity of the gap is destroyed by the pair-breaking effect of time-reversal symmetry breaking perturbations [2, 3], e.g. magnetic impurities [4], and parallel magnetic fields in thin films [5]. (For a general review see, e.g., Refs. [6.7]). A second, and more direct way of influencing the integrity of the gap is through the imposition of quenched spatial fluctuations of the superconducting coupling constant [8, 9]. Physically, such inhomogeneities can be induced by dislocations, twin or grain boundaries, or compositional heterogeneity as found in superconducting alloys [10]. In these cases, fluctuations in the superconducting coupling constant are reflected in inhomogeneities of the order parameter which, in turn, induce a smearing of the quasi-particle energy gap.

In all of the cases described above, the mechanism by which the quasi-particle energy gap is suppressed follows a similar scheme and is described by the same phenomenology: at the mean-field level, each of the perturbations above lead to a suppression of the quasi-particle gap edge. The form of this suppression is contained within the Abrikosov-Gor'kov theory [4] which describes the rearrangement of the ground state under the constraints imposed by the self-consistency equation. While the physical mechanisms of gap suppression differ, the mean-field equations depend on a single dimensionless parameter characterising the strength of the external perturbation (see below). Even at the mean-field level, it is found that if the perturbation is strong enough, the system is driven into a homogeneous gapless phase before the superconductivity is ultimately destroyed.

After the pioneering work of Abrikosov and Gor'kov [4] (originally formulated in the context of magnetic impurities in the dirty superconductor), it was realised that the integrity of the gapped phase is compromised even if the perturbation is weak [11 13]. Optimal fluctuations of the random impurity potentials can conspire to create quasi-particle states localised on the length scale of the coherence length $\xi$ at energies below the mean-field gap, i.e. in the presence of disorder, the system fragments into an inhomogeneous phase in which 'droplets' of localised sub-gap states are embedded in the superconducting background. A similar scenario arises in proximity coupled systems, i.e. SN hybrid structures, where the superconductor induces a gap in the normal region [14,15]. In the most recent investigation, it was shown 13] that, close to the mean-field energy gap edge $E_{\text {gap }}$, the nature of the quasi-particle states (their structure and spectral density) are universal, depending only on the relative separation from the edge, the dimensionality, and the single dimensionless parameter characterising the strength of the perturbation.

On this background, we consider below the influence of a spatially varying coupling constant $g(\mathbf{r})$ on the quasiparticle properties of a conventional disordered $s$-wave superconductor. As mentioned above, such a program is not new: the same problem was investigated in an earlier work by Larkin and Ovchinnikov [8]. However, although our aims, and indeed many of our conclusions, are broadly similar to those of Ref. [8], this investigation is motivated by two considerations: firstly, the development of a quasi-classical approach within the framework of the non-linear $\sigma$-model (NL $\sigma \mathrm{M})$ to explore the nature of the quasi-particle states in the 'sub-gap' region serves as a useful prototype for future studies of related 'droplet phase' instabilities in other interacting theories such as that presented by the superconductor/insulator transition in the disordered interacting system [16, 17]. Secondly, in developing and applying the $\sigma$-model approach, we will find that the Lifshitz-type arguments [18] invoked in Ref. [8] to determine the profile of the DoS in the sub-gap region are flawed. Indeed, the theory developed below will expose a general scheme which establishes the universality of 'gap fluctuations' in the $d$-dimensional system (and which is in accord with the zero-dimensional results of Ref. [14]). 
With this introduction, let us formulate the model superconducting system which we will consider. Our starting point is the Gor'kov or Bogoliubov-de Gennes Hamiltonian

$$
\mathcal{H}=\left(\begin{array}{cc}
\mathcal{H}_{0} & \Delta \\
\Delta & -\mathcal{H}_{0}^{T}
\end{array}\right)_{\mathrm{PH}}
$$

where the subscript ' $\mathrm{PH}$ ' refers to the particle/hole space and $\mathcal{H}_{0}=\mathbf{p}^{2} / 2 m-\epsilon_{\mathrm{F}}+V(\mathbf{r})$. Here, $\epsilon_{\mathrm{F}}$ denotes the Fermi energy and $V(\mathbf{r})$ represents a quenched random impurity potential whose distribution is characterised by the mean scattering time $\tau$. For simplicity, we consider the potential to be drawn from a Gaussian white noise distribution, $\left\langle V(\mathbf{r}) V\left(\mathbf{r}^{\prime}\right)\right\rangle_{V}=\left(2 \pi \nu_{0} \tau\right)^{-1} \delta\left(\mathbf{r}-\mathbf{r}^{\prime}\right)$, where $\nu_{0}$ is the average DoS of the normal system.

The order parameter (chosen to be real) has to be determined self-consistently from the condition $g^{-1}(\mathbf{r}) \Delta(\mathbf{r})=\left\langle\psi_{\uparrow}(\mathbf{r}) \psi_{\downarrow}(\mathbf{r})\right\rangle / \nu_{0}$. Following Ref. [8], we will assume that the (inverse) coupling constant $g^{-1}(\mathbf{r})$ exhibits small fluctuations around an average value $1 / \bar{g}$. As with the random impurity potential, these fluctuations of the coupling constant $\delta(1 / g)(\mathbf{r}) \equiv g_{1}(\mathbf{r})$ are drawn from a Gaussian distribution with zero mean, and correlation

$$
\left\langle g_{1}(\mathbf{r}) g_{1}\left(\mathbf{r}^{\prime}\right)\right\rangle_{g}=\phi\left(\left|\mathbf{r}-\mathbf{r}^{\prime}\right|\right)
$$

(our specific choice of notation being borrowed from Ref. [8]). Here we will impose the condition $g_{1}(\mathbf{r}) \bar{g} \ll 1$ so that the coupling constant remains positive everywhere. Furthermore, we will assume that the correlations are characterised by some correlation length $r_{c}$ which determines the range of $\phi$.

Qualitatively, the response of the ground state to inhomogeneities in the coupling constant depends sensitively on the range of the correlations. If the correlation length $r_{c}$ is much larger than the superconducting coherence length

$$
\xi=\left(\frac{D}{2|\Delta|}\right)^{1 / 2}
$$

(where $D$ diffusion constant), then, the order parameter can smoothly adjust to the local value of $g^{-1}(\mathbf{r})$. In this case $\Delta(\mathbf{r}) \sim g(\mathbf{r})$, and the local DoS, $\nu(\mathbf{r})$, is fixed by the local value of the order parameter [8]. In the opposite limit, one expects the faster fluctuations of the coupling constant to be rectified by the 'proximity effect' coupling of neighbouring superconducting regions. It is in this limit that the system becomes sensitive to quasi-classical phase coherence processes. Therefore, to focus our discussion, in the following, we limit consideration to the quasi-classical and dirty limits, where the energy scales are arranged in the hierarchy

$$
\epsilon_{\mathrm{F}} \gg 1 / \tau \gg \Delta \text {. }
$$

Before turning to the formalism, let us summarise the main conclusions of this investigation. Firstly, as anticipated by Ref. [8], it is found that inhomogeneities of the coupling constant are reflected in inhomogeneities of the superconducting order parameter. Setting $\Delta(\mathbf{r})=$ $\bar{\Delta}+\Delta_{1}(\mathbf{r})$, where $\bar{\Delta}$ represents the homogeneous component of the order parameter and $\Delta_{1}(\mathbf{r})$ its spatial fluctuation, one finds that

$$
\left\langle\Delta_{1}(\mathbf{q}) \Delta_{1}(-\mathbf{q})\right\rangle_{g}=\bar{\Delta}^{2}\left\langle g_{1}(\mathbf{q}) g_{1}(-\mathbf{q})\right\rangle_{g} f^{2}(|\mathbf{q}|) .
$$

Here $f(|\mathbf{q}|)$ represents a dimensionless function which is determined self-consistently (see below).

Now, if the correlation length of the coupling constant is short as compared to the coherence length, $r_{c} \ll \xi$, one finds [8] that the equation of motion for the average quasi-particle Green function obeys a local nonlinear equation which has the canonical form obtained in the theory of gapless superconductivity by Abrikosov and Gor'kov [4]. Specifically, in the mean-field approximation, the BCS singularity is rounded off, and the DoS exhibits a reduced quasi-particle energy gap [8]

$$
E_{\text {gap }}=\bar{\Delta}\left(1-\eta^{2 / 3}\right)^{3 / 2}
$$

where

$$
\eta \sim \phi(0)\left(\frac{r_{c}}{\xi \ln \left(\xi / r_{c}\right)^{2}}\right)^{2}
$$

is a dimensionless parameter characterising the strength of the correlations of the superconducting order parameter. Note that, in the present case, the conditions $g_{1}(\mathbf{r}) \bar{g} \ll 1$ (i.e. $\Delta_{1}(\mathbf{r}) \ll \bar{\Delta}$ ) and $r_{c} \ll \xi$ imply that $\eta \ll 1$. Thus, as one would expect, if the coupling constant remains positive everywhere, the system remains in the gapped phase.

However, the conclusions of the mean-field analysis are modified significantly by optimal fluctuations of the random impurity potential. Such fluctuations, which appear as spatially inhomogeneous instanton configurations of the mean-field equation, show the gap structure to be fragile: mesoscopic fluctuations generate spatially localised states at energies below the mean-field gap. Close to the mean-field gap edge $E_{\text {gap }}$, these states are confined to droplets of size [8]

$$
r_{\text {drop }}(\epsilon) \sim \xi\left(\frac{E_{\text {gap }}-\epsilon}{\bar{\Delta}}\right)^{-1 / 4}
$$

diverging as $\epsilon$ approaches $E_{\text {gap }}$. Since $r_{\text {drop }} \gg \ell \gg \lambda_{\mathrm{F}}$, each of these regions is characterised by an entire band of localised states confined to each droplet. To exponential accuracy the corresponding sub-gap DoS varies as

$$
\nu(\epsilon) \sim \exp \left[-a_{\mathrm{d}}(\eta) \nu_{0} D L^{d-2}\left(\frac{\xi}{L}\right)^{d-2}\left(\frac{E_{\mathrm{gap}}-\epsilon}{\bar{\Delta}}\right)^{\frac{6-d}{4}}\right],
$$

where $a_{\mathrm{d}}(\eta)$ represents a known dimensionless function of the control parameter $\eta$. This result, which is nonperturbative in the dimensionless conductance of the 
normal system $\nu_{0} D L^{d-2}$, differs from that obtained in Ref. [8] but, instead, mirrors the scaling obtained in the study of sub-gap states in superconductors with magnetic impurities [12]. Later we will argue that the energy scaling of the DoS is not accidental but is a universal feature of the sub-gap states in the superconducting system (c.f. Ref. [13]).

When $r_{\text {drop }}>L$, the system enters a zero-dimensional regime. Here the expression for the DoS (2) applies with $d=0$. Reassuringly, this result is found to be in accord with the exact universal result predicted for zerodimensional models which exhibit a square root singularity at the level of mean-field [14]. (The origin of this universality in the present scheme was discussed in Ref. [13].)

The paper is organised as follows: in Sec. II we formulate the quantum partition function of the disordered superconductor in the framework of a replica field theory. In the dirty limit, we show that the low-energy properties of the bulk superconducting system is contained within a non-linear $\sigma$-model action. Taking into account the response of the quasi-particles to inhomogeneities in the BCS coupling constant, we obtain a renormalised action describing gap fluctuations. Within a mean-field analysis we show that the quasi-particle energy gap is suppressed but the integrity of the gap edge is preserved. Taking into account instanton configurations of the action, in section III we show that integrity of the gap edge is compromised. An analysis of the fluctuations in the vicinity of the instanton configurations shows that optimal fluctuations of the impurity potential induce sub-gap states localised on the length scale of the superconducting coherence length. Finally, in Sec. IV we conclude on the universality of the results obtained here.

\section{FIELD THEORY OF THE INHOMOGENEOUS SUPERCONDUCTOR}

The field theory approach to the study of weakly disordered systems [19 21] has been discussed and reviewed extensively in the literature (see, e.g., Ref. [22]). Its extension to the consideration of disordered superconducting systems follows straightforwardly 223, 17, 24]. Therefore, here we will only briefly summarise the main elements in the construction of the (replica) field theory of the disordered superconductor in the framework of the non-linear $\sigma$-model. Using this formulation, we will thereafter investigate the response of the superconducting system to inhomogeneities in the BCS coupling constant.

\section{A. Non-linear $\sigma$-model}

The starting point of the analysis is the coherent state path integral for the replicated partition function [20:21]

$$
\mathcal{Z}^{N}=\int \prod_{a=1}^{N} D \bar{\psi}^{a} D \psi^{a} e^{-\sum_{a=1}^{N} S\left[\psi^{a}\right]},
$$

where $\psi^{a}$ represent Grassmann fields and, defining Matsubara frequencies for the Fermi system, $\epsilon_{n}=(2 n+$ 1) $\pi / \beta$,

$$
S\left[\psi^{a}\right]=\int d \mathbf{r} \sum_{n \sigma} \bar{\psi}_{n \sigma}^{a}\left(i \epsilon_{n}-\mathcal{H}_{0}\right) \psi_{n \sigma}^{a}+S_{I}\left[\psi^{a}\right]
$$

with

$$
S_{I}\left[\psi^{a}\right]=\frac{1}{\nu_{0}} \int_{0}^{\beta} d \tau \int d \mathbf{r} g(\mathbf{r}) \bar{\psi}_{\uparrow}^{a} \bar{\psi}_{\downarrow}^{a} \psi_{\downarrow}^{a} \psi_{\uparrow}^{a} .
$$

To account for the symmetry properties of the system, it is convenient to enlarge the field space by introducing the four-component fields $\Psi^{a T}=\left(\psi_{\uparrow}^{a}, \bar{\psi}_{\downarrow}^{a}, \psi_{\downarrow}^{a},-\bar{\psi}_{\uparrow}^{a}\right) / \sqrt{2}$. This incorporates a particle/hole as well as time-reversal (or charge conjugation) space. Decoupling the quartic BCS interaction with the introduction of the order parameter $\Delta(\mathbf{r})$ (chosen to be real), the total action assumes the canonical form

$$
\begin{aligned}
S\left[\Psi^{a}\right]= & \int d \mathbf{r} \sum_{n} \bar{\Psi}_{n}^{a}\left(i \epsilon_{n} \sigma_{3}^{\mathrm{CC}}-\mathcal{H}\right) \Psi_{n}^{a} \\
& +\nu_{0} \int_{0}^{\beta} d \tau \int d \mathbf{r} g^{-1}(\mathbf{r}), \Delta^{2}(\mathbf{r}, \tau)
\end{aligned}
$$

where $\mathcal{H}$ represents the Gor'kov Hamiltonian defined above (1D). Here $\sigma_{3}^{\mathrm{CC}}$ is a Pauli matrix in the newly introduced charge conjugation space.

To explore the low-energy properties of the superconducting system we follow a standard route: as with normal disordered conductors, when subjected to an ensemble average over the random impurity distribution, the functional integral over the Fermionic fields $\Psi$ can be traded for an integral involving matrix fields $Q$. Physically, the fields $Q$, which vary slowly on the scaling of the mean-free path $\ell$, describe the soft modes of density relaxation - the diffusion modes. In the quasi-classical limit $\left(\epsilon_{\mathrm{F}} \gg 1 / \tau\right)$, the action for $Q$ is dominated by the saddle-point field configuration. In the dirty limit $(1 / \tau \gg \Delta)$, the saddle-point equation

$$
Q(\mathbf{r})=\frac{i}{\pi \nu_{0}}\left\langle\mathbf{r}\left|\left(i \hat{\epsilon} \sigma_{3}^{\mathrm{PH}} \otimes \sigma_{3}^{\mathrm{CC}}+\frac{\partial^{2}}{2 m}+\epsilon_{\mathrm{F}}+\frac{i}{2 \tau} Q\right)^{-1}\right| \mathbf{r}\right\rangle,
$$

where $\sigma_{3}^{\mathrm{PH}}$ is a Pauli matrix in particle/hole space, admits the solution $Q_{0}=\Lambda \otimes \sigma_{3}^{\mathrm{PH}} \otimes \sigma_{3}^{\mathrm{CC}}$, with $\Lambda_{n m}=\operatorname{sgn}\left(\epsilon_{n}\right) \delta_{n m}$ and $[\hat{\epsilon}]_{n m}=\epsilon_{n} \delta_{n m}$. In the limit $\epsilon \rightarrow 0$, the saddle point is not unique but spans an entire manifold parameterised by the unitary transformations $Q=T Q_{0} T^{-1}$. Taking into account slow spatial and temporal fluctuations of the fields, the low-energy long-range properties of the weakly disordered superconducting system are described by a non-linear $\sigma$-model action [22,23, 17,24$]$ 


$$
S[Q, \Delta]=\nu_{0} \int d \mathbf{r} \int_{0}^{\beta} d \tau g^{-1}(\mathbf{r}) \Delta^{2}(\mathbf{r}, \tau)+\frac{\pi \nu_{0}}{8} \int d \mathbf{r} \operatorname{tr}\left[D(\partial Q)^{2}-4\left(\hat{\epsilon} \sigma_{3}^{\mathrm{PH}} \otimes \sigma_{3}^{\mathrm{CC}}+\Delta \sigma_{2}^{\mathrm{PH}}\right) Q\right],
$$

where the matrix field obeys the non-linear constraint $Q^{2}=\mathbb{1}$. The Hermitian matrices $Q$ obey the the symmetry relation $Q=\sigma_{1}^{\mathrm{PH}} \otimes \sigma_{1}^{\mathrm{CC}} Q^{T} \sigma_{1}^{\mathrm{PH}} \otimes \sigma_{1}^{\mathrm{CC}}$ reflecting the symmetry properties of the dyadic product $\Psi \otimes \bar{\Psi}$. Note that the fields carry replica $(a, b)$ as well as Matsubara $(n, m)$ indices, i.e. $Q=Q_{n m}^{a b}$.

This completes the formulation of the disordered superconducting system as a functional field integral involving the replicated $\mathrm{NL} \sigma \mathrm{M}$ action. Our interest here is in the thermodynamic DoS obtained as

$$
\nu(\epsilon)=-\pi^{-1} \int \frac{d \mathbf{r}}{V} \Im\left[G\left(\mathbf{r}, \mathbf{r} ; i \epsilon_{n} \rightarrow \epsilon^{+}\right)\right] .
$$

Making use of the analytic continuation $\ln \mathcal{Z}=$ $\lim _{N \rightarrow 0}\left(\mathcal{Z}^{N}-1\right) / N$, it is straightforward to show that the impurity averaged DoS can be obtained from the identity

$$
\langle\nu(\epsilon)\rangle=\frac{\nu_{0}}{4} \int \frac{d \mathbf{r}}{V} \lim _{N \rightarrow 0}\left\langle\operatorname{tr}\left[\Lambda \otimes \sigma_{3}^{\mathrm{PH}} \otimes \sigma_{3}^{\mathrm{CC}} Q P_{\epsilon \epsilon}\right]\right\rangle_{Q, \Delta},
$$

where $\langle\cdots\rangle_{Q, \Delta}=\int D Q \int D \Delta \cdots e^{-S[Q, \Delta]}$ and $P_{\epsilon \epsilon}$ projects onto the diagonal element $\epsilon \epsilon$.

\section{B. Self-consistent fluctuations of the order parameter}

Following Ref. [8], our strategy will be to use the meanfield solution of the homogeneous problem as a platform to develop a perturbative expansion of the self-consistent order parameter. Specifically, by finding the deviation $\delta \Delta(\mathbf{r})=\Delta_{1}(\mathbf{r})$ of the order parameter from its mean value $\bar{\Delta}$ to leading order in $g_{1}(\mathbf{r})$, integrating out fast fluctuations of $Q$, and averaging over random configurations of $g_{1}(\mathbf{r})$, we will obtain an effective action for the quasi-particle degrees of freedom of the superconducting system. With this effective theory, we will again use a saddle-point analysis to explore the rearrangement of the ground state due to the inhomogeneous coupling constant. At the mean-field level, the solution reveals a homogeneous renormalisation of the superconducting gap from its bare value. On this background, we will find that the hard gap predicted by the mean-field theory is further softened by gap fluctuations which are accommodated in the effective field theory by inhomogeneous instanton configurations of the fields $Q$.

Following this program, we begin by subjecting the action to a saddle-point analysis. Varying the action (3) with respect to $Q$ and $\Delta$, one obtains the coupled saddlepoint equations

$$
\begin{aligned}
& D \partial(Q \partial Q)+\left[Q, \hat{\epsilon} \sigma_{3}^{\mathrm{PH}} \otimes \sigma_{3}^{\mathrm{CC}}+\Delta \sigma_{2}^{\mathrm{PH}}\right]=0 \\
& g^{-1}(\mathbf{r}) \Delta(\mathbf{r})=\frac{\pi}{4 \beta} \operatorname{tr}\left[\sigma_{2}^{\mathrm{PH}} Q(\mathbf{r})\right]
\end{aligned}
$$

For a homogeneous coupling constant, these equations admit a homogeneous solution for the order parameter and $Q$. However, for a general inhomogeneous configuration for $g^{-1}(\mathbf{r})$, an exact solution is unavailable and an approximate scheme must be sought.

Applied to the saddle-point equations (5) above, the Ansatz

$$
Q_{n m}=\left(\cos \hat{\theta}_{n} \sigma_{3}^{\mathrm{PH}} \otimes \sigma_{3}^{\mathrm{CC}}+\sin \hat{\theta}_{n} \sigma_{2}^{\mathrm{PH}}\right) \delta_{n m},
$$

where $\hat{\theta}_{n}=\operatorname{diag}\left(\theta_{n}^{1}, \ldots, \theta_{n}^{N}\right)$ is replica diagonal, leads to the coupled saddle-point equations

$$
\begin{aligned}
& D \partial^{2} \hat{\theta}_{n}(\mathbf{r})-2\left(\epsilon_{n} \sin \hat{\theta}_{n}(\mathbf{r})-\Delta(\mathbf{r}) \cos \hat{\theta}_{n}(\mathbf{r})\right)=0, \\
& g^{-1}(\mathbf{r}) \Delta(\mathbf{r})=\frac{\pi}{\beta} \sum_{n} \sin \hat{\theta}_{n}(\mathbf{r}) .
\end{aligned}
$$

In the following we will drop the Matsubara indices and only reinstate them when necessary. These equations can be identified as self-consistent Usadel equations [25,26] for the average quasi-classical Green function in the presence of an inhomogeneous coupling constant. Specifically, the former represents the reorganisation of the ground state due to spatial inhomogeneities in the order parameter, while the second equation enforces the self-consistency condition imposed on the order parameter.

For a homogeneous coupling constant $\bar{g}$, the mean-field equations are solved by a homogeneous replica symmetric Ansatz with

$$
\hat{\theta}_{0}=\arccos \left(\frac{\hat{\epsilon}}{\hat{E}}\right)
$$

where $\hat{E}^{2}=\hat{\epsilon}^{2}+\bar{\Delta}^{2}$ and $\bar{\Delta}=(\pi \bar{g} / \beta) \sum_{n} \sin \theta_{0 n}$. In this case, as expected, we simply recover the BCS solution 24. This result, being independent of disorder, is simply a manifestation of the Anderson theorem [1] on the level of the effective action - in the non-interacting system, a weak non-magnetic impurity potential has no influence on the average DoS.

To accommodate spatial fluctuations of the coupling constant and, with them, fluctuations of the order parameter one should, in principle, solve the non-linear set of equations self-consistently. Evidently, such a program 
is infeasible. Instead, following Ref. [8], taking the relative fluctuations of the coupling constant to be small, we look for a perturbative expansion of the mean-field equations. To develop the expansion, we set $\hat{\theta}=\hat{\theta}_{0}+\hat{\theta}_{1}(\mathbf{r})$ and, accordingly, $\Delta=\bar{\Delta}+\Delta_{1}(\mathbf{r})$, where both $\hat{\theta}_{1}$ and $\Delta_{1}$ are of order $g_{1}$. Expanding to first order in $g_{1}$, one obtains the coupled linear equations for $\hat{\theta}_{1}$ and $\Delta_{1}$,

$$
\begin{aligned}
& D \partial^{2} \hat{\theta}_{1}-2 \underbrace{\left(\hat{\epsilon} \cos \hat{\theta}_{0}+\bar{\Delta} \sin \hat{\theta}_{0}\right)}_{\hat{E}} \hat{\theta}_{1}+2 \Delta_{1} \cos \hat{\theta}_{0}=0, \\
& \Delta_{1}(\mathbf{r})=\frac{\pi \bar{g}}{\beta} \sum_{n} \theta_{1 n}(\mathbf{r}) \cos \theta_{0 n}-g_{1}(\mathbf{r}) \bar{g} \bar{\Delta} .
\end{aligned}
$$

Transforming Eq. (8a) to the Fourier representation, one obtains the solution

$$
\hat{\theta}_{1}(\mathbf{q})=2 \frac{\Delta_{1}(\mathbf{q}) \cos \hat{\theta}_{0}}{D \mathbf{q}^{2}+2 \hat{E}}
$$

which, when inserted into Eq. 8b, yields

$$
\Delta_{1}(\mathbf{q})=-\frac{\bar{\Delta}}{\frac{\pi}{\Delta \beta} \sum_{n}\left(\sin \theta_{0 n}-\frac{2 \bar{\Delta} \cos ^{2} \theta_{0 n}}{D \mathbf{q}^{2}+2 E_{n}}\right)} g_{1}(\mathbf{q}) .
$$

Finally, performing the Matsubara summation, one finds

$$
\Delta_{1}(\mathbf{q}) \equiv-\bar{\Delta} g_{1}(\mathbf{q}) f(|\mathbf{q}|),
$$

where, normalising the wavevector $\tilde{\mathbf{q}}=\xi \mathbf{q}$ by the coherence length,

$$
\begin{aligned}
f(q) & =\frac{2 \tilde{q}^{2}}{\pi-\left(\tilde{q}^{4}-1\right)^{1 / 2} \ln \left[\frac{\tilde{q}^{2}-\left(\tilde{q}^{4}-1\right)^{1 / 2}}{\tilde{q}^{2}+\left(\tilde{q}^{4}-1\right)^{1 / 2}}\right]} \\
& = \begin{cases}1-\frac{\pi \tilde{q}^{2}}{4}+\ldots & \tilde{q} \ll 1, \\
\frac{1}{\ln \tilde{q}^{2}} & \tilde{q} \gg 1 .\end{cases}
\end{aligned}
$$

From this result, we obtain the response of the order parameter to spatial variations of the BCS coupling constant. As expected, the low Fourier components $(q \ll$ $1 / \xi)$ of the order parameter smoothly follow spatial fluctuations of $g^{-1}(\mathbf{r})$. Perhaps more surprising is the response of the order parameter to fast fluctuations. As one would expect, these fluctuations are suppressed by the proximity effect. However, as noted by Ref. [8], the attenuation scales only as $1 / \ln \tilde{q}^{2}$.

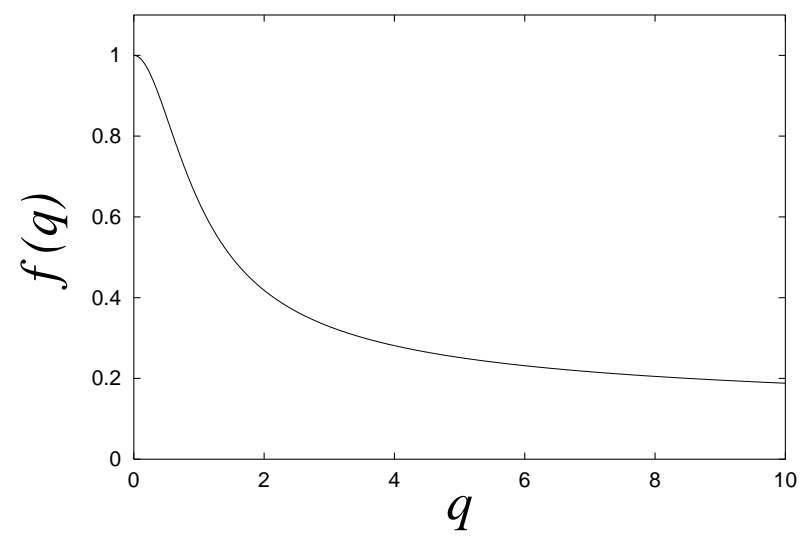

FIG. 1. The function $f(q)$, governing the dependence of the order parameter on variations of the coupling constant.

Now, these fast fluctuations of the order parameter can have a dramatic effect on the mean-field DoS and its fluctuation in the vicinity of the mean-field gap edge. To assimilate the effect of these fluctuations it is necessary to revisit the $\mathrm{NL} \sigma \mathrm{M}$ action taking into account the inhomogeneous order parameter.

\section{Mean-field solution}

Substituting the mean-field solution for the order parameter, $\bar{\Delta}$, together with its spatial fluctuation $\Delta_{1}(\mathbf{r})$ into the $\mathrm{NL} \sigma \mathrm{M}$ action, one obtains

$$
S[Q]=\frac{\pi \nu_{0}}{8} \int d \mathbf{r} \operatorname{tr}\left[D(\partial Q)^{2}-4\left(\hat{\Sigma}+\Delta_{1}(\mathbf{r}) \sigma_{2}^{\mathrm{PH}}\right) Q\right],
$$

where $\hat{\Sigma}=\hat{\epsilon} \sigma_{3}^{\mathrm{PH}} \otimes \sigma_{3}^{\mathrm{CC}}+\bar{\Delta} \sigma_{2}^{\mathrm{PH}}$.

Now, since $\int d \mathbf{r} \Delta_{1}(\mathbf{r})=0$, contributions to the generating function arising from field configurations of $Q$ which are constant or slowly varying in space are largely insensitive to the fluctuations. Therefore, to assess the influence of the spatial inhomogeneity of the order parameter, we proceed by integrating out fast fluctuations of $Q$ [27], where 'fast' means varying on length scales shorter than the coherence length. To do so, the fast and slow degrees are separated by expanding $Q$ around the slowly varying $\bar{Q}$, i.e.

$$
Q=T \bar{Q} T^{-1}, \quad \bar{Q}=e^{-W</ 2} \Lambda \otimes \sigma_{3}^{\mathrm{PH}} \otimes \sigma_{3}^{\mathrm{CC}} e^{W</ 2},
$$

where $T=\exp \left[-W_{>} / 2\right]$ with $\bar{Q} W_{>}+W_{>} \bar{Q}=0$. Integrating over $W_{>}$, one obtains $S_{\text {eff }}=S_{0}+S_{\mathrm{AG}}$, where

$$
S_{0}=\frac{\pi \nu_{0}}{8} \int d \mathbf{r} \operatorname{tr}\left[D(\partial \bar{Q})^{2}-4 \hat{\Sigma} \bar{Q}\right]
$$

and 


$$
S_{\mathrm{AG}}=\frac{\pi \nu_{0}}{8} \sum_{\mathbf{q}, \mathbf{q}^{\prime}, \mathbf{q}^{\prime \prime}, \mathbf{q}^{\prime \prime \prime}} \operatorname{tr}\left[\Delta_{1}(\mathbf{q}) \Delta_{1}\left(-\mathbf{q}^{\prime \prime \prime}\right) \hat{\Pi}_{\mathbf{q}^{\prime},-\mathbf{q}^{\prime \prime}}\left[\bar{Q}\left(\mathbf{q}+\mathbf{q}^{\prime}\right), \sigma_{2}^{\mathrm{PH}}\right]\left[\bar{Q}\left(-\mathbf{q}^{\prime \prime}-\mathbf{q}^{\prime \prime \prime}\right), \sigma_{2}^{\mathrm{PH}}\right]\right] .
$$

Here $\Delta_{1}$ is determined by Eq. (9), and $\hat{\Pi}_{\mathbf{q},-\mathbf{q}^{\prime}}$ represents the diffusion propagator, i.e. $\hat{\Pi}_{\mathbf{q},-\mathbf{q}^{\prime}}^{-1}=D \mathbf{q}^{2} \delta_{\mathbf{q}, \mathbf{q}^{\prime}}+\{\hat{\Sigma}, \bar{Q}(\mathbf{q}-$ $\left.\left.\mathbf{q}^{\prime}\right)\right\}$. Retaining only the diagonal part $\hat{\Pi}_{\mathbf{q},-\mathbf{q}}$ and averaging over fluctuations $g_{1}$, which to a good approximation amounts to replacing $g_{1}(\mathbf{q}) g_{1}\left(-\mathbf{q}^{\prime}\right)$ by its average value $\left\langle g_{1}(\mathbf{q}) g_{1}\left(-\mathbf{q}^{\prime}\right)\right\rangle_{g}=\phi(|\mathbf{q}|) \delta_{\mathbf{q}, \mathbf{q}^{\prime}}$, one obtains

$$
S_{\mathrm{AG}}=\frac{\pi \nu_{0} \bar{\Delta}}{16} \int d \mathbf{r} \int d \mathbf{r}^{\prime} \operatorname{tr}\left[\hat{\eta}\left(\mathbf{r}-\mathbf{r}^{\prime}, \hat{\epsilon}\right)\left[\bar{Q}(\mathbf{r}), \sigma_{2}^{\mathrm{PH}}\right]\left[\bar{Q}\left(\mathbf{r}^{\prime}\right), \sigma_{2}^{\mathrm{PH}}\right]\right]
$$

with

$$
\hat{\eta}(\mathbf{r}, \hat{\epsilon})=\frac{2}{\bar{\Delta}}\left\langle\Delta_{1}^{2}\right\rangle(\mathbf{r}) \hat{\Pi}(\mathbf{r}) .
$$

Upon approaching the gap edge, $\hat{\Pi}$ becomes long-ranged, the relevant scale being $\ell_{E}=\sqrt{D / E} \gg \xi$. Thus, the spatial dependence of $\eta$ is governed by $\left\langle\Delta_{1}^{2}\right\rangle$, whose range is determined by Eq. (9) and the correlator $\left\langle g_{1}(\mathbf{r}) g_{1}\left(\mathbf{r}^{\prime}\right)\right\rangle_{g}=$ $\phi\left(\left|\mathbf{r}-\mathbf{r}^{\prime}\right|\right)$. If $\phi$ is short-ranged (on the scale of the coherence length), we can use the approximation

$$
S_{\mathrm{AG}}=\frac{\pi \nu_{0} \bar{\Delta}}{16} \int d \mathbf{r} \operatorname{tr}\left(\hat{\eta}(0, \hat{\epsilon})\left[\bar{Q}(\mathbf{r}), \sigma_{2}^{\mathrm{PH}}\right]^{2}\right) .
$$

Close to the gap, where the energy dependence of $\hat{\eta}$ is negligible, one has

$$
\hat{\eta}=\eta \simeq \frac{1}{\xi^{2}} \int \frac{d \mathbf{q}}{q^{2}} f^{2}(|\mathbf{q}|) \phi(|\mathbf{q}|) .
$$

In this approximation, we will show below that the action recovers the mean-field equation obtained in Ref. [8].

To summarise, quenched inhomogeneities in the coupling constant induce spatial fluctuations of the order parameter which are accommodated by a rearrangement of the quasi-particles in the superconducting condensate. In the disordered system, this rearrangement is governed by the same Usadel equation that describe the proximity effect in hybrid SN systems. Taking into account the inhomogeneities in the order parameter, one obtains an effective action for the disordered superconductor in which the bulk action for the non-disordered system is supplemented by an additional term (10) which, as we will see presently, leads to a suppression of the superconducting quasi-particle gap.

To explore the influence of the fluctuations on the quasi-particle gap structure let us again vary the action with respect to $\bar{Q}$. In doing so, we obtain the modified saddle-point equation

$$
D \partial(\bar{Q} \partial \bar{Q})+[\bar{Q}, \hat{\Sigma}]-\frac{1}{2} \bar{\Delta} \eta\left[\bar{Q}, \sigma_{2}^{\mathrm{PH}} \bar{Q} \sigma_{2}^{\mathrm{PH}}\right]=0 .
$$

Adopting the parameterisation (6), this saddle-point equation can be rewritten as

$$
D \partial^{2} \hat{\theta}-2[\hat{\epsilon} \sin \hat{\theta}-\bar{\Delta} \cos \hat{\theta}+\bar{\Delta} \eta \sin \hat{\theta} \cos \hat{\theta}]=0 .
$$

The saddle-point equation (11) has a form which coincides with the Abrikosov-Gor'kov (AG) equation obtained in the theory of gapless superconductivity [ 1 . There, the parameter $\eta$ has to be interpreted as the spin scattering rate $\eta=1 / \tau_{s} \bar{\Delta}$ induced by magnetic impurities in the superconducting system. The analysis of the AG equation shows that, for $\eta>1$ the system enters a gapless phase while for $\eta<1$ the quasi-particle energy gap is suppressed but not destroyed.

More precisely, taking the mean-field solution to be homogeneous in space, i.e. $\hat{\theta}(\mathbf{r}) \equiv \hat{\theta}_{\mathrm{AG}}$, the saddle-point equation (11) takes the form

$$
\hat{\epsilon} \sin \hat{\theta}_{\mathrm{AG}}-\bar{\Delta} \cos \hat{\theta}_{\mathrm{AG}}+\bar{\Delta} \eta \sin \hat{\theta}_{\mathrm{AG}} \cos \hat{\theta}_{\mathrm{AG}}=0 .
$$

Combined with the gap equation, the solution is obtained self-consistently from the equation

$$
\frac{\hat{\epsilon}}{\bar{\Delta}}=\cot \hat{\theta}_{\mathrm{AG}}\left(1-\eta \frac{1}{\sqrt{1+\cot ^{2} \hat{\theta}_{\mathrm{AG}}}}\right),
$$

which coincides with Eq. (18) of Ref. [8]. Making use of the Eq. (4), one obtains the mean-field DoS

$$
\nu(\epsilon)=\nu_{0} \Re\left[\cos \theta_{\mathrm{AG}}\left(i \epsilon_{n} \rightarrow \epsilon\right)\right],
$$

which reveals a reduction of the energy gap according to $E_{\text {gap }}=\bar{\Delta}\left(1-\eta^{2 / 3}\right)^{3 / 2}$. In particular, for $\eta<1$, the meanfield theory still predicts a hard gap in the quasi-particle DoS, displaying square root behaviour as $\epsilon \rightarrow E_{\text {gap }}[$ []]:

$$
\nu(\epsilon) \simeq\left\{\begin{array}{cl}
0 & \epsilon<E_{\text {gap }} \\
\nu_{0} \eta^{-2 / 3}\left(1-\eta^{2 / 3}\right)^{-1 / 4} \sqrt{\frac{2}{3} \frac{\epsilon-E_{\text {gap }}}{\Delta}} & \epsilon>E_{\text {gap }}
\end{array}\right.
$$

As expected, quenched disorder in the coupling constant is reflected in an overall suppression of the quasiparticle energy gap. Importantly, quasi-classical processes lead to a suppression of the gap that does not simply follow the distribution of the order parameter. However, as recognised by Larkin and Ovchinnikov [8], the square root singularity in the DoS predicted by the mean-field theory is untenable: optimal fluctuations associated with the impurity potential $V(\mathbf{r})$ must give rise to sub-gap states which cause the gap to fluctuate. How 
are such states accommodated by the statistical field theory?

At first sight it is tempting to seek gap fluctuations within the perturbative fluctuations around the symmetric mean-field saddle-point configuration $\theta_{\mathrm{AG}}$. However, when taken into account, it is found that the integrity of the gap is maintained by the analytical properties of the mean-field solution: perturbative fluctuations influence only the profile of the DoS about the mean-field energy gap. (For a discussion of this point in the context of the hybrid SN system, see Ref. 24]). Instead, to explore states below the mean-field energy gap, it is necessary to revisit the saddle-point equation (11) and seek inhomogeneous 'symmetry breaking' instanton field configurations.

\section{INHOMOGENEOUS SADDLE POINTS: STRATEGY}

To develop a theory of sub-gap states in the present system we can draw intuition both from the analysis of Larkin and Ovchinnikov [8] as well as a related study of gap fluctuations in the superconductor with magnetic impurities [12]. In Ref. [8] sub-gap states were shown to be associated with inhomogeneous solutions of the Abrikosov-Gor'kov equation (11). In the framework of the field theory of the non-interacting system, these inhomogeneous instanton or bounce solutions break supersymmetry at the level of the action. In the present case, we can therefore anticipate that the relevant bounce configurations break the replica symmetry, providing an exponential suppression of the DoS below the mean-field edge. At the same time, we expect to identify a zeromode in the replica space which restores the global replica symmetry of the theory.

In the following, for simplicity, we will first restrict attention to the quasi one-dimensional system and describe later how the result can be generalised to higher dimensions.

\section{A. Replica symmetry breaking}

In order to investigate inhomogeneous solutions, we first have to understand the structure of the homogeneous solution in more detail. The Fermionic integration contour covers the interval $\theta \in[0, \pi]$. As the mean-field DoS vanishes below the gap edge (i.e. $\nu(\epsilon)=\nu_{0} \Re[\cos \theta]=0$ ), one knows that the mean-field solution of the AbrikosovGor'kov equation satisfies the condition $\theta_{\mathrm{AG}}\left(\epsilon<E_{\mathrm{gap}}\right)=$ $\pi / 2+i \phi_{\mathrm{AG}}(\epsilon)$, where $\phi_{\mathrm{AG}}(\epsilon)$ is real.

Now instead of analysing the saddle-point equation, it is more convenient to study its first integral

$$
\xi^{2}(\partial \theta)^{2}+V(\theta)=\text { const. }
$$

where, switching from Matsubara to real energies, the effective potential is given by

$$
V(\theta)=-2 i \frac{\epsilon}{\bar{\Delta}} \cos \theta+2 \sin \theta+\frac{\eta}{2} \cos 2 \theta
$$

Along the line $\theta=\pi / 2+i \phi$ the potential $V_{R}(\phi)=$ $-V(\pi / 2+i \phi)$ is real with a functional dependence on $\phi$ shown in Fig. 2 for different energies. The homogeneous saddle point sits at the maximum of this potential. At $\epsilon=0$, it belongs to the contour $\left(\theta_{\mathrm{AG}}=\pi / 2\right)$ and the potential is symmetric around $\phi=0$. By increasing the energy, the saddle point moves away from the real axis along the line $\theta_{\mathrm{AG}}=\pi / 2+i \phi_{\mathrm{AG}}$ until the energy reaches $E_{\text {gap }}$. On this line the mean-field DoS vanishes. At energies $\epsilon>E_{\text {gap }}$ the real part of $\theta_{\mathrm{AG}}$ starts to deviate from $\pi / 2$ and the DoS becomes finite. Thus, even at the mean-field level the integration contour must be smoothly deformed to reach the saddle-point solution (for a discussion see, e.g., Ref. [24]). Following the behaviour of the potential, one notices that one minimum deepens while the other becomes more and more shallow until merging with the maximum at $\epsilon=E_{\text {gap }}$.

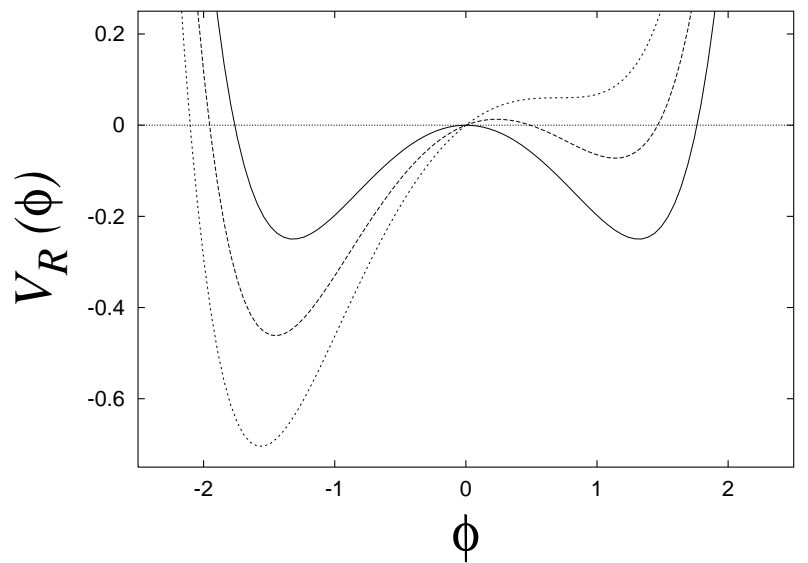

FIG. 2. Real potential as a function of $\phi$ along the line $\theta=\pi / 2+i \phi$ for $\epsilon=0, E_{\text {gap }} / 2, E_{\text {gap }}$. At $\epsilon=E_{\text {gap }}$ the maximum and the minimum merge.

Now, in addition to the homogeneous AbrikosovGor'kov solution, the potential above admits for a bounce solution $\phi_{\mathrm{AG}} \rightarrow \phi_{\max }\left(>\phi_{\mathrm{AG}}\right) \rightarrow \phi_{\mathrm{AG}}$, where $V_{R}\left(\phi_{\max }\right)=$ $V_{R}\left(\phi_{\mathrm{AG}}\right)$. In principle, as is clear from Fig. 2, this is not the only inhomogeneous solution. However, a bounce solution towards negative values of $\phi$ always involves a larger action and its contribution can therefore be neglected. For $\Re[\theta] \neq \pi / 2$ the imaginary part of the potential is finite, in general. 


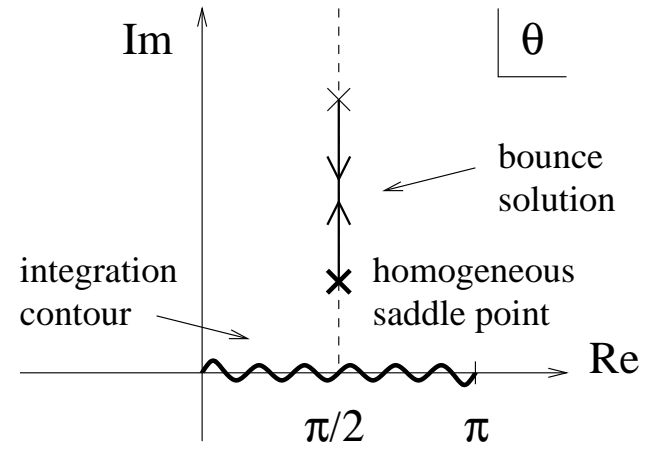

FIG. 3. Bounce solution.

In order to obtain a finite though exponentially small sub-gap density of states, we need to find a solution with finite action. All replica symmetric solutions lead to a vanishing action in the limit $N \rightarrow 0$. Therefore, the solution we seek necessarily involves replica symmetry breaking (RSB). Leaving aside the homogeneous mean-field configuration, the configuration which incurs the lowest action is one in which the bounce inhabits only a single replica, say, $a=1$, i.e.

$$
\hat{\theta}(x)=\frac{\pi}{2}+i \operatorname{diag}\left(\phi(x), \phi_{\mathrm{AG}}, \ldots, \phi_{\mathrm{AG}}\right) .
$$

In general, an analytic solution of the highly non-linear saddle-point equation is not available. However, close to the gap edge, one can expand $V_{R}(\phi)$ around the homogeneous solutions at $E_{\text {gap }}$ up to cubic order:

$$
\begin{aligned}
& V_{R}(\phi)-V_{R}\left(\phi_{\mathrm{AG}}\right) \\
\simeq & -\sqrt{6}\left(\frac{E_{\text {gap }}}{\bar{\Delta}}\right)^{1 / 6} \gamma^{1 / 2}(\epsilon) \delta \phi^{2}+\left(\frac{\eta E_{\text {gap }}}{\bar{\Delta}}\right)^{1 / 3} \delta \phi^{3},
\end{aligned}
$$

where $\gamma(\epsilon)=\left(E_{\text {gap }}-\epsilon\right) / \bar{\Delta}$ and $\delta \phi=\phi-\phi_{\mathrm{AG}}$. In this limit the solution can be obtained analytically, namely

$$
\delta \phi(x)=\sqrt{6}\left(\frac{\bar{\Delta}}{\eta^{2} E_{\mathrm{gap}}}\right)^{1 / 6} \gamma^{1 / 2}(\epsilon) \cosh ^{-2}\left(\frac{x}{2 r_{\mathrm{drop}}(\epsilon)}\right) .
$$

Similarly, the action for the instanton assumes the form

$$
S_{\text {inst. }}=4 \pi \nu_{0} \bar{\Delta} \xi \int_{\phi_{\mathrm{AG}}}^{\phi_{\max }} d \phi \sqrt{V_{R}\left(\phi_{\mathrm{AG}}\right)-V_{R}(\phi)} .
$$

The size of the instanton, which diverges upon approaching the gap edge, is given by [8]

$$
r_{\text {drop }}(\epsilon)=6^{1 / 4} \xi\left(\frac{\bar{\Delta}}{E_{\text {gap }}}\right)^{1 / 12} \gamma^{-1 / 4}(\epsilon) .
$$

The inhomogeneous solution, thus, varies on length scales much longer than the coherence length justifying the separation into slow and fast degrees of freedom in Sec. II O.
In higher dimensions one can assume that the bounce solution possesses radial symmetry. Nevertheless, the problem becomes more complicated because the saddlepoint equation contains a gradient term [28]

$$
\partial_{\tilde{r}}^{2} \phi+\frac{d-1}{\tilde{r}} \partial_{\tilde{r}} \phi+\frac{1}{2} \partial_{\phi} V_{R}(\phi)=0
$$

where $\tilde{r}=|\mathbf{r}| / \xi$.

However, one can still determine the parameter dependence of the action by dimensional analysis using the Ansatz $\delta \phi=\alpha f(|\mathbf{r}| / b)$. Altogether, this obtains

$$
S_{\text {inst. }}=a_{\mathrm{d}}(\eta) \nu_{0} D \xi^{d-2} \gamma(\epsilon)^{(6-d) / 4},
$$

where $a_{\mathrm{d}}(\eta)=c_{\mathrm{d}} \eta^{-2 / 3}\left(1-\eta^{2 / 3}\right)^{-(2+d) / 8}$ and $c_{\mathrm{d}}$ a numerical constant; $c_{1}=2^{7} \pi \sqrt{6} / 5$.

Although this completes our analysis of the profile and statistical weight of the bounce solution, since it does not depart from the line $\Re[\theta]=\pi / 2$, taken alone, it provides no contribution to the DoS! To understand why sub-gap states are associated with the bounce it is necessary to explore the role of fluctuations in the vicinity of the instanton. As emphasised in Ref. [12], such a program turns out to be crucial in the present system.

\section{B. Fluctuation analysis}

As we have seen, the lowest energy bounce configuration involves a breaking of replica symmetry at the level of the mean-field. Taking into account fluctuations in the vicinity of the bounce solution, we will see below that there exists a zero-mode and a negative energy mode. The former restores global replica symmetry of the theory and, thus, ensures the integrity of the normalisation of the generating functional $\mathcal{Z}=1$. Furthermore, the negative energy mode necessitates a $\pi / 2$ rotation of the contour which, imparting a factor of $i$, renders the contribution of the instanton to the DoS non-vanishing.

To explore the influence of the fluctuations, let us return to the quasi one-dimensional system and introduce the parameterisation

$$
Q=R e^{-W / 2} \Lambda \otimes \sigma_{3}^{\mathrm{PH}} \otimes \sigma_{3}^{\mathrm{CC}} e^{W / 2} R^{-1},
$$

where $R(x)=\exp \left[i \sigma_{1}^{\mathrm{PH}} \otimes \sigma_{3}^{\mathrm{CC}} \theta(x) / 2\right]$ is the rotation from the metallic saddle point to the inhomogeneous saddle point $Q_{\mathrm{SP}}(\theta)$. The matrices $W$ are subject to the symmetry condition in replica space $W_{b a}=W_{a b}^{\dagger}$. Expanding the action up to second order in the generators obtains the following term:

$$
S[W] \simeq-\frac{\pi \nu_{0}}{8} \int d \mathbf{r} \sum_{a b} \operatorname{tr}\left[\partial W_{a b} \partial W_{b a}+\frac{1}{2} \partial \phi_{a} \partial \phi_{b} \sigma_{2}^{\mathrm{PH}} W_{a b} \sigma_{2}^{\mathrm{PH}} W_{b a}+\left(F_{a}-\eta \cosh \phi_{a} \cosh \phi_{b}\right) W_{a b} W_{b a}\right.
$$




$$
\left.-\eta \sinh \phi_{a} \sinh \phi_{b} \sigma_{1}^{\mathrm{PH}} \otimes \sigma_{3}^{\mathrm{CC}} W_{a b} \sigma_{1}^{\mathrm{PH}} \otimes \sigma_{3}^{\mathrm{CC}} W_{b a}\right],
$$

where $F_{a}=\left(\left(\partial \phi_{a}\right)^{2}+V\left(\phi_{a}\right)-\eta \cosh 2 \phi_{a}\right) / 2$. There are two types of fluctuation:
(a) replica-diagonal fluctuations, and
(b) fluctuations mixing the replicas.

Within the replica-diagonal part, the most relevant contributions are due to fluctuations of the angle $\phi$, i.e. $W_{a b}=\sigma_{1}^{\mathrm{PH}} \otimes \sigma_{3}^{\mathrm{CC}} \varphi_{a}(x) \delta_{a b}$. In the $N-1$ 'trivial' replicas $\left(\theta=\theta_{\mathrm{AG}}\right)$, these fluctuations are massive and, therefore, only lead to a weakly energy dependent prefactor. More important are the fluctuations which stay within the symmetry broken replica $a=1$ :

$$
S\left[\varphi_{1}\right]=\frac{1}{2} \int d x \int d x^{\prime} \varphi_{1}(x) \frac{\delta^{2} S}{\delta \phi(x) \delta \phi\left(x^{\prime}\right)} \varphi_{1}\left(x^{\prime}\right) .
$$

Now this class of fluctuations has been studied extensively in the standard literature 29. The operator $\delta^{2} S / \delta \phi(x) \delta \phi\left(x^{\prime}\right)$ has a zero-mode, $\varphi_{1}^{(0)} \sim \partial \phi$, due to translational invariance, i.e. the action is independent of the position of the bounce. Furthermore, as the zeromode is associated with a bounce solution it has a node. This implies the existence of one negative energy eigen mode. To account for this, one has to rotate the contour away from the imaginary axis. This deformation of the contour provides a factor of $i$. Therefore, the result which was purely imaginary $(\Re[\theta]=\pi / 2)$ before - becomes real and, thus, gives a finite contribution to the DoS.

Turning to the fluctuations mixing the replicas, the replica symmetry breaking must be accompanied by a zero-mode in replica space. Writing $W=W^{-}+W^{+}$, where $W^{-}\left(W^{+}\right)$(anti-)commutes with $\sigma_{1}^{\mathrm{PH}} \otimes \sigma_{3}^{\mathrm{CC}}$, the part of the action involving the coupling between the replicas ' 1 ' and ' $a \neq 1$ ' reads

$$
S_{1 a}^{ \pm} \sim-\sum_{a \neq 1} \operatorname{tr}\left[\partial W_{1 a}^{ \pm} \partial W_{a 1}^{ \pm}+\frac{V[\phi(\mathbf{r})]-\widetilde{V}^{ \pm}}{2} W_{1 a}^{ \pm} W_{a 1}^{ \pm}\right],
$$

where

$$
\widetilde{V}^{ \pm}=\frac{\eta}{2}\left(\cosh 2 \phi_{\mathrm{AG}}+\cosh 2 \phi(\mathbf{r})+4 \cosh \left(\phi_{\mathrm{AG}} \pm \phi(\mathbf{r})\right)\right) .
$$

Although its presence is disguised by the choice of parameterisation, the action involving this class of fluctuations exhibits a zero-mode. Indeed, its existence is made manifest by specifying the parameterisation $Q=$ $U Q_{\mathrm{SP}} U^{\dagger}$. However, with this choice, the measure associated with the fluctuations becomes highly non-trivial. Nevertheless, it is useful for determining the dependence of the integration over zero-modes on the number of replicas, $N$. Close to the gap edge, i.e. at finite energies $\epsilon>0$, the structure of the saddle point within the PHand CC-space is completely fixed. - The only freedom left are rotations in replica space, $U \in \mathrm{U}(N)$. Thus, dividing off the matrices which leave the saddle point invariant, the relevant matrices $U$ belong to the coset space $\mathrm{U}(N) /(\mathrm{U}(1) \times \mathrm{U}(N-1))$. Integration over the zero-mode gives a factor which is proportional to the volume $\sim N$ in the limit $N \rightarrow 0$ [30]. Or, in other words, there are $N$ saddle points that contribute to the integral. Using the parameterisation (14) does not change the $N$ dependence but only the spatial structure of the zero-mode. Therefore, without calculating the value of the prefactor, we know that the result has the following form:

$$
\begin{aligned}
& \nu(\epsilon) \sim \lim _{N \rightarrow 0} \frac{1}{N} \int d x N\left[\sinh \phi(x)+(N-1) \sinh \phi_{\mathrm{AG}}\right] \times \\
& \times\left|\chi_{0}(x)\right|^{2} e^{-S_{\text {inst. }}} \\
& =\int d x\left[\sinh \phi(x)-\sinh \phi_{\mathrm{AG}}\right]\left|\chi_{0}(x)\right|^{2} e^{-S_{\text {inst. }}},
\end{aligned}
$$

where $\left|\chi_{0}(x)\right|^{2}$ describes the spatial profile of the zeromode.

\section{Discussion}

This concludes our derivation of the sub-gap DoS: by itself the instanton or bounce configuration provides the leading exponential dependence of the DoS while the fluctuations render the pre-exponential factors positive definite. More precisely,

(1) the prefactor becomes real due to the negative energy eigen mode and the consequential deformation of the contour;

(2) the sub-gap DoS is non-vanishing only in the vicinity of the bounce as can be seen from Eq. (15).

Altogether, taking the expression for the action in the $d$-dimensional system (13), we obtain the expression for the DoS defined by Eq. (2). A further rescaling of the DoS allows an explicit connection between the general expression and its universal zero-dimensional limit: to this end, let us introduce the parameter

$$
\Delta_{\mathrm{g}}=\left(\frac{2}{3} \bar{\Delta} \delta^{2}\right)^{1 / 3} \eta^{4 / 9}\left(1-\eta^{2 / 3}\right)^{1 / 6}
$$

where $\delta=1 /\left(\nu_{0} V\right)$ is the average level spacing of the normal system, after which the mean-field DoS in the vicinity of the energy gap can be cast in the form 14. 


$$
\nu\left(\epsilon>E_{\text {gap }}\right)=\frac{1}{\pi V} \sqrt{\frac{\epsilon-E_{\text {gap }}}{\Delta_{\mathrm{g}}^{3}}} .
$$

Then, making use of Eqs. (16) and (12), the DoS can be brought to the more compact form

$$
S_{\text {inst. }}=\sqrt{\frac{3}{2}} c_{\mathrm{d}}\left(\frac{r_{\mathrm{drop}}(\epsilon)}{L}\right)^{d} \gamma_{\mathrm{g}}(\epsilon)^{3 / 2},
$$

where $\gamma_{\mathrm{g}}(\epsilon)=\left(E_{\text {gap }}-\epsilon\right) / \Delta_{\mathrm{g}}$ and $r_{\text {drop }}$ is defined in Eq. (12). Indeed, the factor $\left(r_{\mathrm{drop}} / L\right)^{d}$ can be further absorbed into $\Delta_{\mathrm{g}}$ by replacing the level spacing of the system, $\delta$, with the level spacing of the droplet, $\delta_{\text {drop }}(\epsilon)=1 /\left(\nu_{0} r_{\text {drop }}^{d}\right)$, in its definition (16). Now, as noted in Ref. [14], if the mean-field DoS exhibits a squareroot singularity of the form (17), fluctuations of the edge due to optimal fluctuations of the impurity potential are predicted to assume a universal form

$$
\nu(\epsilon) \sim \exp \left[-\frac{2}{3}\left(\frac{E_{\mathrm{gap}}-\epsilon}{\Delta_{\mathrm{g}}}\right)^{3 / 2}\right]
$$

obtained by random matrix theory. Now, as we have seen, when $r_{\text {drop }}>L$ (inevitable as $\epsilon \rightarrow E_{\text {gap }}$ ), the system enters a zero-dimensional regime. In this limit, with $d=0$ the expression for the DoS reassuringly assumes the universal form (19).

In the present context, the mechanism by which the universal expression develops at the level of the action has been elucidated in Ref. 13]. Specifically, in the zerodimensional regime, the instanton configuration must be supplemented by a homogeneous replica symmetry broken solution of stationary phase which sits at the shallow minimum of the potential $V(\theta)$, c.f. Fig. 2. There the integration contour leaves the axis $\theta=\pi / 2+i \phi$, and the minimum represents in fact a maximum along the perpendicular direction [13]. Physically, gap fluctuations in the zero-dimensional system correspond to sample-tosample fluctuations rather than spatial variations of the gap.

Although the saddle-point analysis as well as the size of the instanton agree with the result found in Ref. [], the energy dependence of the action does not, i.e. while we obtain the exponent $\alpha=3 / 2-d / 4$, the solution obtained in Ref. [8] is compatible with an exponent $\alpha_{\text {Lif }}=2-d / 4$. As mentioned above the exponent $3 / 2$ can be traced back to the zero-dimensional case, where the form of the action is universal [14], i.e. this exponent is a direct consequence of the square root behaviour of the mean-field result.

In fact, the discrepancy of the results can be traced to the application of a Lifshitz-type argument [18] to the present scheme. Indeed, although the problem bears close similarity to the Lifshitz problem of band tail states in semiconductors 18, 31, the correspondence is superficial. In particular, Lifshitz tails states at the band-edge of a semiconductor are typically associated with wavefunctions which vary smoothly on the scale of their extent. As such, an estimate of the optimal character of the tail state distribution can be established on the level of the $\psi$-field action. By contrast, the sub-gap tail states associated with gap fluctuations in the superconducting system involve a superposition of states close to the Fermi level, where spatial fluctuations vary rapidly oscillating at the scale of the Fermi wavelength - the sub-gap states are quasi-classical in origin. It therefore does not seem possible to develop a Lifshitz argument for the present system. As a further consequence, in contrast to the band-tail states, the quasi-classical nature of the subgap states in the superconductor make their properties insensitive to the nature of the impurity distribution.

\section{CONCLUSIONS}

In summary, following the work of Larkin and Ovchinnikov [8], we have shown that, in a weakly disordered superconductor, short-scale fluctuations of the BCS coupling constant lead to a suppression of the quasi-particle energy gap. At the level of mean-field, the integrity of the gap edge is maintained. However, optimal fluctuations of the impurity potential induce a narrow band of states, localised at the scale of the coherence length, which extend below the mean-field gap edge. Within the framework of the statistical field theory developed here, these states appear as replica symmetry broken instanton configurations of the mean-field equations - the global symmetry of the theory being restored by a zero-mode in the replica space. To exponential accuracy, we have obtained the spectrum of gap fluctuations. The generality of these results has been emphasised. Specifically, in the $d$-dimensional system, once normalised by the mean-field DoS at the gap edge, we have shown that the spectrum of tails states depends only on the dimensionless parameter $\eta$ and, in particular, is independent of the nature of the disorder potential. Moreover, in the zero-dimensional system, the spectrum of gap fluctuations is truly universal and coincides with that obtained by Vavilov et al. 14. in the study of gap fluctuations in the SN system.

Finally, it is interesting to note that the analysis in this work has a number of relatives in the recent literature. As we have emphasised, at the level of the soft mode action, the theory of gap fluctuations mirrors that obtained in the Abrikosov-Gor'kov theory of a superconductor with magnetic impurities [12,13] and, later, that encountered in the description of sub-gap states in the hybrid SN system [15]. Furthermore, various results non-perturbative in the (inverse) dimensionless conductance and involving replica (or super-)symmetry breaking have been reported in the literature [32 35]. Of these investigations, it is particularly interesting to contrast the present scheme with the prediction of 'anomalously localised states' in the weakly disordered normal conductor.

By exploiting instanton configurations of the non-linear $\sigma$-model action, Khmel'nitskii and Muzykantskii [33] proposed that the long-time current 
relaxation in a disordered wire was dominated by rare localised states which coexist in a background of extended states (see also, Ref. 34). These states, which are ascribed to optimal fluctuations of the random potential, are penalised by a statistical weight which depends exponentially on the dimensionless conductance. This scaling mirrors that found in the present system. However, crucially, scaling in the superconducting system involves an energy dependence which allows the exponent to become small as one approaches the energy gap.

In hindsight, it is easy to understand why optimal fluctuations can more readily induce localised states in the superconducting system. In the normal disordered system, as pointed out by Mott, hybridisation makes the coexistence of localised states in a background of extended states difficult to sustain. However, in the superconducting system, fluctuations of the order parameter provide a natural mechanism by which quasi-particle states can localise in regions where the order parameter is suppressed.

ACKNOWLEDGEMENTS: We are grateful to Dima Khmel'nitskii and Austen Lamacraft for useful discussions.

[1] P. W. Anderson, J. Phys. Chem. Sol. 11, 26 (1959).

[2] K. Maki, Prog. Theor. Phys. (Kyoto) 29, 333 (1963); 31, 731 (1963); 32, 29 (1964); K. Maki and P. Fulde, Phys. Rev. 140, A1586 (1965).

[3] P. G. de Gennes, Phys. Kondens. Materie 3, 79 (1964).

[4] A. A. Abrikosov and L. P. Gor'kov, Sov. Phys. JETP 12, 1243 (1961).

[5] Yu. N. Ovchinnikov, Sov. Phys. JETP 29, 853 (1969).

[6] K. Maki, "Gapless Superconductivity" in R. D. Parks (ed.), Superconductivity, vol. II, Dekker, New York (1969), Chapter 18.

[7] P. G. de Gennes, Superconductivity of Metals and Alloys, W. A. Benjamin, New York 1966; M. Tinkham, Introduction to Superconductivity, 2nd edition, McGraw-Hill, New York 1996.

[8] A. I. Larkin and Yu. N. Ovchinnikov, Sov. Phys. JETP 34, 1144 (1972).

[9] L. B. Ioffe and A. I. Larkin, Sov. Phys. JETP 54, 378 (1981).
[10] A. A. Abrikosov, Fundamental Theory of Metals, NorthHolland, Amsterdam 1988.

[11] A. V. Balatsky and S. A. Trugman, Phys. Rev. Lett. 79, 3767 (1997).

[12] A. Lamacraft and B. D. Simons, Phys. Rev. Lett 85, 4783 (2000).

[13] A. Lamacraft and B. D. Simons, preprint condmat/0101080.

[14] M. G. Vavilov, P. W. Brouwer, V. Ambegaokar, and C. W. J. Beenakker, Phys. Rev. Lett. 86, 874 (2001).

[15] P. M. Ostrovsky, M. A. Skvortsov, and M. V. Feigelmann, preprint cond-mat/0012478.

[16] Yu. N. Ovchinnikov, Sov. Phys. JETP 37, 366 (1973).

[17] A. M. Finkel'stein, JETP Lett. 45, 46 (1987); Physica B 197, 636 (1994).

[18] I. M. Lifshitz, Sov. Phys. Usp. 7, 549 (1965).

[19] F. J. Wegner, Z. Phys. B 35, 207 (1979).

[20] K. B. Efetov, A. I. Larkin, and D. E. Khmel'nitskii, Sov. Phys. JETP 52, 568 (1980).

[21] A. M. Finkel'stein, Sov. Phys. JETP 57, 97 (1983).

[22] K. B. Efetov, Supersymmetry in Disorder and Chaos, Cambridge University Press, New York 1997.

[23] R. Oppermann, Nucl. Phys. B 280, 753 (1987); V. E. Kravtsov and R. Oppermann, Phys. Rev. B 43, 10865 (1991).

[24] A. Altland, B. D. Simons and J. P. D. Taras-Semchuk, JETP Lett. 67, 22 (1998); Adv. Phys. 49, 321 (2000).

[25] K. D. Usadel, Phys. Rev. Lett. 25, 507 (1970); Phys. Rev. B 4, 99 (1971).

[26] A. I. Larkin and Yu. N. Ovchinnikov, J. Low. Temp. Phys. 10, 407 (1973).

[27] See e.g. Ref. 22, pp. 257/8.

[28] A. Zee and I. Affleck, J. Phys. Cond. Matt. 12, 8862 (2000).

[29] See, e.g., S. Coleman, in Aspects of Symmetry, Cambridge University Press, 1985.

[30] A. Kamenev and M. Mezard, J. Phys. A 32, 4373 (1999); Phys. Rev B 60, 3944 (1999).

[31] B. I. Halperin and M. Lax, Phys. Rev. 148, 722 (1966); J. Zittartz and J. S. Langer, Phys. Rev. 148, 741 (1966)

[32] K. B. Efetov and V. G. Marikhin, Phys. Rev. B 40, 12126 (1989).

[33] B. A. Muzykantskii and D. E. Khmel'nitskii, Phys. Rev. B 40, 12126 (1989).

[34] V. I. Fal'ko and K. B. Efetov, Phys. Rev. B 52, 17413 (1995); Europhys. Lett. 32, 627 (1995).

[35] A. Kamenev, Phys. Rev. Lett. 85, 4160 (2000). 